\title{
Local illness concepts and their relevance for the prevention and control of malaria during pregnancy in Ghana, Kenya and Malawi: findings from a comparative qualitative study
}

Arantza Menaca ${ }^{1,2}$, Christopher Pell ${ }^{1,3^{*}}$, Lucinda Manda-Taylor ${ }^{4}$, Samuel Chatio ${ }^{5}$, Nana A Afrah ${ }^{6}$, Florence Were ${ }^{7}$, Abraham Hodgson ${ }^{5}$, Peter Ouma ${ }^{7}$, Linda Kalilani ${ }^{4}$, Harry Tagbor ${ }^{6}$ and Robert Pool ${ }^{1,3}$

\begin{abstract}
Background: In sub-Saharan Africa, the burden of morbidity and mortality linked to malaria during pregnancy (MiP) is significant and compounded by its unclear symptoms and links with other health problems during pregnancy. Mindful of the biomedical and social complexity of MiP, this article explores and compares local understandings of MiP and their links with other pregnancy-related health problems.

Methods: A comparative qualitative study was undertaken at four sites in three countries: Ghana, Malawi and Kenya. Individual and group interviews were conducted with pregnant women, their relatives, opinion leaders, other community members and health providers. MiP-related behaviours were also observed at health facilities and in local communities.

Results: Across the four sites, local malaria concepts overlapped with biomedically defined malaria. In terms of symptoms, at-risk groups, outcomes and aetiology of malaria during pregnancy, this overlap was however both site-specific and partial. Moreover, the local malaria concepts were not monolithic and their descriptions varied amongst respondents. The symptoms of pregnancy and malaria also overlapped but, for respondents, symptom severity was the distinguishing factor. Malaria was generally, though not universally, perceived as serious for pregnant women. Miscarriage was the most widely known outcome, and links with anaemia, low birth weight and congenital malaria were mentioned. Nonetheless, amongst many potential causes of miscarriage, malaria was not recognized as the most important, but rather interacted with other pregnancy-related problems.

Conclusions: Given the overlap of common pregnancy problems with the symptoms of malaria, and the limited association of malaria with its main outcomes, a comprehensive antenatal care programme is the most appropriate strategy for the provision of health education, prevention and treatment for MiP. Variations in locally shared understandings of MiP must however be taken into account when designing and promoting MiP intervention strategies.
\end{abstract}

Keywords: Malaria, Pregnancy, Qualitative methods, Local disease concepts, Africa

\footnotetext{
*Correspondence: c.l.pell@uva.nl

'Centre de Recerca en Salut Internacional de Barcelona (CRESIB), Hospital

Clínic-Universitat de Barcelona, Barcelona, Spain

${ }^{3}$ Centre for Social Science and Global Health, University of Amsterdam,

Amsterdam, The Netherlands

Full list of author information is available at the end of the article
} 


\section{Background}

In endemic regions of sub-Saharan Africa, malaria in pregnancy (MiP) is a major preventable cause of maternal morbidity and poor birth outcomes [1]. Current recommended strategies for MiP in sub-Saharan Africa include appropriate case management, intermittent preventive treatment (IPTp) with sulphadoxine-pyrimethamine (SP), and insecticide-treated bed nets (ITNs) [2]. These strategies, however, must be delivered in a context-specific way that takes into account changing levels of transmission and drug resistance [3]. Nonetheless, despite the efforts and the progress made over the last decade, the coverage of IPTp and ITNs amongst pregnant African women is still inadequate [4]. In response to the recognized burden of MiP-related morbidity and mortality and to address the ongoing challenges of prevention and control, the MiP Consortium, comprised of 47 partner institutions in 32 countries, is currently conducting a wide range of research activities in Africa, Asia and Latin America [5]. This article draws on the results of an anthropological programme of research that forms part of the consortium's Public Health Impact group.

The overall goal of the MiP Consortium's anthropological programme is to contribute to the development of appropriate MiP interventions by gaining an in-depth understanding of MiP-related attitudes and behaviours at an individual and social level. A review of previous research [6] identified four broad topics that influence the uptake of MiP interventions: concepts of malaria and risk in pregnancy; attitudes towards malaria prevention and treatment; perceptions of antenatal care (ANC) services; and structural factors. These four themes have been widely explored by the MiP Consortium's anthropology programme and this article focuses on the local concepts of malaria and risk in pregnancy. Additional articles will focus on attitudes towards malaria prevention and treatment interventions, and perceptions of ANC [7]. The analysis of the structural factors affecting delivery and uptake of MiP interventions is however integral to all these articles.

To ensure a thorough analysis of their impact on health-seeking practices, local illness concepts must be studied together with their relationship with biomedical models of disease. Biomedical understandings of MiP are particularly complex and this is apparent across three domains, which are all relevant for health-seeking behaviour: symptoms, risk groups and disease outcomes.

\section{Symptoms}

Although in areas of intense stable transmission MiP has been commonly considered asymptomatic, studies have shown that it is often symptomatic [8] and its symptoms are non-specific - history of fever, headache, malaise, arthromyalgias, vomiting and fatigue. Therefore a blood test (optical microscopy or rapid diagnostic test (RDT)) is necessary to confirm diagnosis [9].

\section{Risk groups}

Not all pregnant women in Africa are equally vulnerable to MiP. In areas of high, stable malaria transmission, $\mathrm{MiP}$ is more prevalent and associated with worse outcomes among women experiencing their first or second pregnancy (frequently adolescents) [1,10]. However, in low, unstable transmission areas, MiP is usually symptomatic, acute and affects all parities equally [8].

\section{Outcomes}

Multiple medical studies have demonstrated that MiP is associated with severe maternal anaemia, pregnancy loses, low birth weight (LBW), congenital malaria and perinatal and infant mortality $[1,11]$. More than one quarter of cases of severe anaemia and one fifth of LBW cases are attributable to malaria in areas of stable high malaria transmission [1]. Except in the case of congenital malaria, which is rarely associated with clinical disease $[12,13]$, all the other outcomes are not univocally associated with malaria: there are a range of different aetiological factors for anaemia in pregnancy [14-16], stillbirths [17], LBW [18] and perinatal and infant mortality $[19,20]$.

The biomedical complexity of MiP - and particularly the often multifactorial relationship between the disease, its symptoms and outcomes - therefore poses challenges for health education and appropriate disease management. Furthermore, in many settings, malaria is also a socially and culturally complex disease. Indeed, previous qualitative research has demonstrated that, all over Africa, local understandings of malaria are varied and often diverge from the biomedical model [21]. A number of studies have also illustrated how these understandings are widely influenced by contact with biomedical health services and health education [22,23]. The anthropological research has shown that local understandings of diseases are generally not systematic, closed nor static: they depend on experiences and change over the time. Moreover, they are not monolithic or consistent among different members of a population [22,24,25]. Anthropological literature also demonstrates that local health concepts cannot be viewed in isolation, but rather that they are related to one another in semantic and experiential networks [22,26]. It is, therefore, necessary to direct attention to how MiP is associated with its symptoms and effects, and to study MiP in the context of general understandings of pregnancy [6].

Mindful of both the biomedical and social complexity of MiP, this article analyses local perceptions of malaria and risk during pregnancy with the objective of identifying: 1) the most (culturally) appropriate framework for 
the design and implementation of MiP interventions, and 2) contradictions between biomedical and local understandings of MiP that could be addressed in health education.

\section{Methods}

The findings presented in this article are drawn from a comparative qualitative study at four sites in three different countries. A multidisciplinary team (made up of social scientists and biomedically trained researchers) based in Ghana, Malawi, Kenya and Spain carried out the study.

\section{Settings}

The study incorporated one country from each of the three main regions of sub-Saharan Africa: Ghana in West Africa, Kenya in East Africa and Malawi in Southern Africa. Two sites with important regional specificities were selected in Ghana for several reasons: to collect data in at least one site of each of the MiP Consortium's main treatment and prevention activities; to include areas with different patterns of malaria transmission; and to examine intra- as well as inter-country variation.

In central Ghana, fieldwork was conducted in two districts of the Ashanti Region: Ejisu Juaben and Ahafo Ano South. In both districts, agriculture is the main productive activity, and there is a significant proportion of internal migrants, in addition to the majority ethnic group, the Asante [27]. At this site, malaria transmission is moderately high and occurs throughout the year with peaks during the rains in May to October [28]. In each district, data collection was conducted at the district hospitals, two to three health centres, and several smaller clinics.

In northern Ghana, Upper East Region, the fieldwork sites were located in Kassena-Nankana District. This area is part of the Sahel and experiences only one annual rainy season during which people grow millet, maize and vegetables for subsistence. During the rest of the year, part of the population migrates to other regions. The Kassena and the Nankani make up almost $90 \%$ of the local population [27]. Here, malaria transmission is hyperendemic: transmission takes place year-round but there is a seasonal pattern with a peak that coincides with the major rains (May to October) and low rates of infection during the dry season [29]. Data were collected at a district hospital in Navrongo, the district capital, and outreach community-based services, which are common throughout the area.

Fieldwork also took place in Chikwawa and Blantyre Districts, southern Malawi. The main ethnic groups in Blantyre District are Chewa and Yao, whereas, in Chikwawa District, they are Manganja and Sena. Most of the women in the area cultivate crops for subsistence and sale at the market. Both districts are in areas of intense, year-round malaria transmission [30]. Fieldwork took place at three hospitals, and six healthcare centres that provide ANC services to the women of these areas.

Finally, in Kenya, fieldwork was carried out in Siaya District, Nyanza Province, where the principal ethnic group, the Luo, make up over $95 \%$ of the population. Livelihood activities include subsistence farming of maize, sorghum, millet and cassava. Due to the relatively limited employment opportunities, migration to urban centres is common, particularly to Kisumu, the nearest city. Malaria is endemic and transmission year-round [31] with the greatest disease burden borne by children and pregnant women. Data were collected at the district hospital and smaller health facilities where ANC is delivered.

\section{Data collection}

An anthropological approach was taken to data collection and this entailed a long phase of fieldwork, a wide range of data collection activities, including free-listing and sorting, in-depth interviews, focus group discussion and participant observation, the use of narrative and observational tools, and a flexible, reflexive and iterative process of tool design, data collection and analysis.

Fieldwork was carried out between April 2009 and August 2011, and lasted from one year in Malawi to more than two years in central Ghana. Fieldworkers spent extended periods of time in the communities where data were collected and recorded their experiences of participant observation in field diaries. Participant observation entailed various activities, which depended on the context: for example, in health care facilities, this often involved sitting with the women waiting for consultations, observing interactions with health staff and informally chatting, whereas, in the communities, this could entail assisting with basic chores. In the first phase, at each site, using free-listing and sorting exercises, the research team explored the main problems that pregnant women experience. Later interviews and group discussions were conducted, several women (case studies) were followed and interviewed several times over the course of their pregnancies (see Table 1 for further details), and observations were carried out in the communities and at local health facilities. The research team interacted with informants in their chosen language (English and various local languages). At all sites, in-depth interviews and group discussions were recorded, transcribed verbatim and then (if necessary) translated into English by fieldworkers.

Communication between staff based at the research sites and in Barcelona was frequent and complemented by quarterly field visits. During these visits, members of the research team reflected on the data collection process, discussing at length preconceptions, unexpected findings, contradictions, doubts and fieldwork dilemmas. 
Table 1 Respondents

\begin{tabular}{|c|c|c|c|c|c|c|}
\hline Method & Respondent type & Central Ghana & Northern Ghana & Kenya & Malawi & Total \\
\hline \multirow[t]{3}{*}{ Free listing and sorting } & Community members & 12 & 16 & 17 & 24 & 59 \\
\hline & Pregnant women & 10 & 10 & 7 & 11 & 38 \\
\hline & Health providers* & 10 & 11 & 6 & 5 & 32 \\
\hline \multirow[t]{4}{*}{ In-depth interviews } & Pregnant women & 84 & 64 & 69 & 68 & 285 \\
\hline & Health providers* & 33 & 34 & 17 & 21 & 105 \\
\hline & Relatives & 26 & 29 & 20 & 16 & 91 \\
\hline & Opinion leaders & 12 & 12 & 10 & 12 & 46 \\
\hline Case studies & Pregnant women & 19 & 18 & 12 & 18 & 67 \\
\hline Focus group discussions & Community members & 10 & 16 & 9 & 16 & 51 \\
\hline
\end{tabular}

*Includes health care staff involved with the provision of ANC at health facilities and TBA working in the communities.

The quarterly field visits also allowed the Barcelona based social science team to participate in data collection and provide on-going training.

Using a range of data collection tools in combination with the flexible, iterative and reflexive research approach meant that perceptions of the relationship between pregnancy and malaria could be explored from several perspectives. Interviews started with broad research questions related to pregnancy, later, they focused on the problems and diseases that concern pregnant women, and ended with questions about MiP. In contrast, focus group discussions started with general questions about malaria, focusing later on groups particularly vulnerable to malaria and finalizing with MiP. Miscarriage, stillbirths, pre-term deliveries, birth weight and anaemia - and their causes - were also themes explored during interviews. Data collection and analysis were carried out in parallel allowing the incorporation of emerging themes in the design of the tool, and questions' .

Several measures were taken to reduce the impact of potential sources of bias and ensure the reliability of findings. To limit the possible influence of individual researchers on the study results, data collection was carried out by at least two team members at each site (a fieldworker and social scientist in central Ghana and Kenya, and, due to higher staff turnover, four fieldworkers and a social scientist in Malawi, and five fieldworkers and a social scientist in northern Ghana). Moreover, employing a range of data collection techniques ensured that findings could be triangulated. By carrying out interviews with a variety of respondents (Table 1) and observations in a range of local contexts (healthcare facilities and community spaces), a range of perspectives were explored and incorporated into the study findings.

\section{Respondents}

Five main categories of participants were interviewed (Table 1): pregnant women, their relatives, community members (men and women), opinion leaders and healthcare providers. Purposive sampling was used to ensure that respondents with a wide range of experiences participated in the study. Married and unmarried pregnant women of a range of ages (under 18 to over 60 years), parities (zero to over seven) and gestational ages (zero to nine months) from across the different communities (within the field sites) were interviewed. Relatives included mainly mothers, mothers-in-law and husbands of the pregnant women. The sample of opinion leaders was made up of religious leaders, traditional and political authorities, and women in the local communities. Finally, ANC staff, pharmacists and drug sellers, traditional birth attendants and other relevant healers were interviewed at each site. Respondents were identified in ANC clinics and via contacts in the local communities that strengthened as fieldwork progressed. The final number of participants was a result of the directed sampling and the point of saturation, whereby no further novel insights were identified from interviews.

\section{Data analysis}

At each site, a first phase of data analysis ran in parallel to data collection. Using Atlas.ti 6, flexible codebooks were developed and revised using a combination of established categories based on the original research questions and themes that emerged from the data. The preliminary results obtained from this site-specific analysis were compared and discussed amongst the members of the team in periodic meetings throughout data collection. In a second phase, data associated to the codes relevant to malaria in pregnancy perceptions, were extracted, collated and discussed between authors one and two, looking at the similarities, differences and variations between and within the different sites.

\section{Ethics statement}

Overall ethics clearance was obtained from the Clinical Research Ethics Committee, Hospital Clinic-University of Barcelona. Separate local ethics clearance was obtained at each site: in Ghana, clearance was obtained from the Institutional Review Board of the Navrongo Health Research 
Centre, Navrongo and the Committee on Human Research Ethics, Kwame Nkrumah University of Science \& Technology, Kumasi; in Kenya, clearance was obtained from the Institution Review Board of Centers for Disease Control and Prevention, Atlanta and from the National Ethics Review Committee, Kenya Medical Research Institute, Nairobi; and in Malawi, clearance was obtained from the College of Medicine Research and Ethics Committee. As approved by all ethics review committees and institutional review boards, informed consent was obtained orally from study participants. Oral rather than written informed consent was obtained because the study procedures posed minimal risk to study participants and to avoid the possible negative influence of a written consent on rapport between researchers and respondents. With the agreement of participants, oral consent was recorded prior to each interview or focus group.

\section{Results}

\section{Terms, symptoms and causes}

In the context of pregnancy, the meanings of local terms that were translated as "malaria" or used to describe malaria-related illnesses coincided only partially with biomedically defined malaria. Hence in some instances, respondents described having malaria, which health professionals did not consider to be malaria. At other times however health professionals diagnosed malaria, which the pregnant woman did not recognize as such because it did not coincide with her understanding of the overlapping local illness concept. In this article, italicized malaria refers to these locally defined illness concepts across the four sites - encapsulating the specific causation, symptoms and outcomes associated with each illness - and malaria (not italicized) to refer to the biomedically defined disease. The terms used to describe these various malarias, as opposed to the concept as a whole, are placed in speech marks (except in quotations).

The terms used to label illnesses that overlapped with malaria originated from local languages and English and, at each site, more than one term was often used. In Malawi, and in northern Ghana respondents mainly used the local terms "malungo" (Chichewa), "paa" (Kassem) and "poa" (Nankam). At both sites, although less frequently, the terms "malaria" and "fever" were also used. In Kenya and central Ghana, the situation was the reverse: respondents generally made use of the English words "malaria" (in Kenya and central Ghana) and "fever" (in central Ghana). In central Ghana, a minority of respondents used local language terms: "whuraye" (whiteness), or occasionally "ebunu" (greenish vomit) or "tiridi" (yellowish eyes).

These terms were used to describe illnesses with a range of symptoms, such as fever, vomiting (generally producing liquid of a yellowish colour), weakness/ fatigue, general body pain, headache, coldness/shaking, joint pains, abdominal/stomach pains, diarrhoea, loss of appetite, dizziness, yellow eyes/urine/faeces, a bitter taste, thirst, paleness, breathlessness, swollen legs, and rashes/itching. The majority of these symptoms were common across the four sites and coincide with the (albeit broad) clinical description of malaria. Others, such as rashes or itching, are not recognized as clinical symptoms of malaria but were reported in northern and central Ghana, and also in Kenya where one respondent diagnosed her own "malaria" based on one such a symptom alone.

Some symptoms were specific to particular local malarias. For example, breathlessness in Kenya; paleness in central Ghana; swollen legs (among the Nankam) and abdominal pains (among the Kassem) in northern Ghana. Across the sites, no specific symptoms were described for pregnant women compared to other segments of the population. However, vomiting in northern and central Ghana and Kenya, and weakness in both Ghana sites played more prominent roles during pregnancy. Given the wide variety of symptoms associated with malaria, respondents in Ghana and Malawi explained that the experiences depended on each individual:

I (interviewer): The poa that you talked of: if someone has malaria, how does she/he know that she/he has poa?

R3 (respondent 3): When you feel dizzy and you also vomit, it means that you have malaria. At times, you may vomit yellow, which tells you that you have poa. (...)

R2: $\quad$ As for poa, we all experience it in a different way because we don't all have the same system. There are some who have itches on their bodies when they have poa. In addition to that, some don't vomit, they rather defecate yellowish but watery faeces.

I: What do you have to say about that madam?

R4: $\quad$ Yes, what I want to say is that poa comes in different forms. There are some people that, when they begin to have it, they can't vomit but feel very cold and have stomach pains.

(Northern Ghana. Focus group discussion with female community members)

Figure 1 provides a visual representation of how the various local illnesses overlapped with malaria (and pregnancy) in terms of the symptoms experienced. In an 


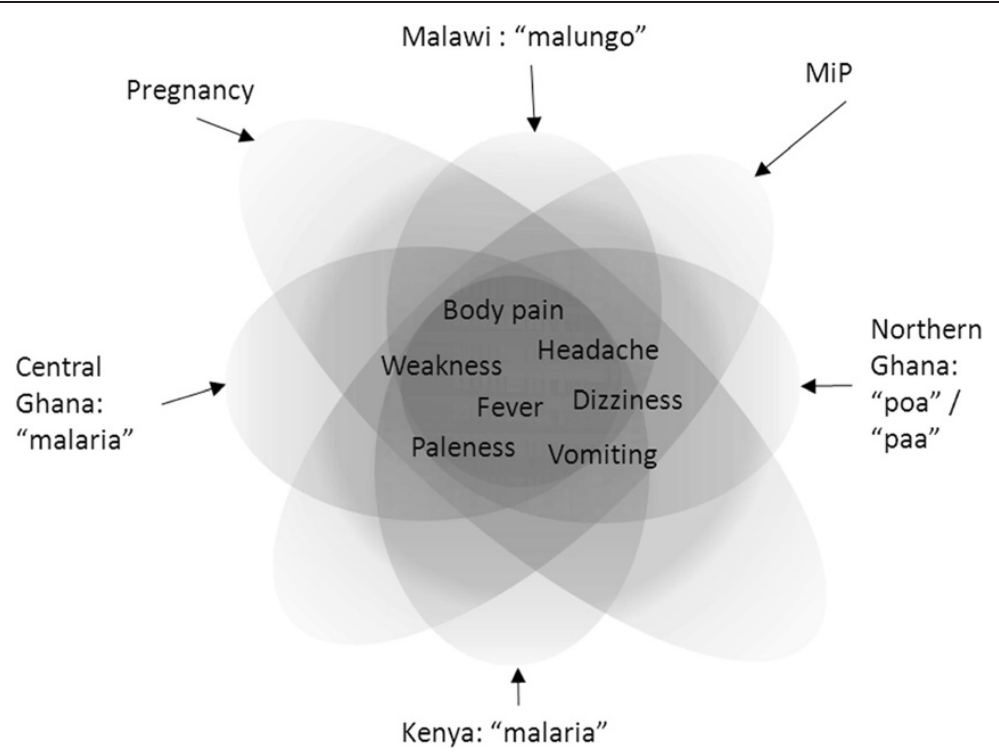

Figure 1 The overlapping symptoms of local malarias, biomedically defined malaria and pregnancy.

abstract manner, the area of each shape represents the range of symptoms that were attributed to each illness and the symptoms most commonly mentioned across the sites are listed in the central overlapping area. In light of the varied descriptions of each illness across the different sites, and the reports of the individual nature of symptoms, this is inevitably a simplification. To encapsulate some of the variation and uncertainty that surrounded the illnesses, each shape is opaque.

At every site, mosquitoes were identified as the main cause of each locally defined illness that overlapped with malaria. Respondents also frequently mentioned poor hygiene and, in some cases, there was a clear link between a lack of cleanliness and mosquitoes, such as stagnant water, bushes around the compound, mosquitoes on the walls, but, in others, there was no such link: dirty cooking utensils, uncovered food and houseflies, cold food and/or, untreated drinking water. At all the sites, a minority of women however reported not knowing the cause of the various illnesses that overlapped with malaria. Exposure to cold and rain was occasionally considered to influence one's chances of getting "malaria" in Kenya and "malaria" or "fever" in central Ghana, and Malawian respondents also associated hard work with "malungo". Some food types were also mentioned as causes of the "poa" or "paa" in Ghana: some vegetables and, especially, sweet foods in the north and oily and spicy food at the central site.

At three of the four sites (not Kenya), pregnancy was also generally seen as a direct contributory factor to bouts of malaria (see Figure 2). For some informants, pregnant women experience these illnesses (and sickness in general) if they neglect the pregnancy: missing ANC appointments and ignoring health workers' advice in Ghana and Malawi; or not using pregnancy-specific traditional medicine in central Ghana.

Pregnancy brings about a lot of things. It can give you headache and you sneeze. You feel different in your body. You might vomit. The vomiting is there, but every [type of] vomit means something and yellowish vomit means malaria. Maybe you feel a sour taste in your mouth: this is malaria. I did not have this before I was pregnant, but now I have all these symptoms. I sneeze, and cough, and vomit yellowish, and after eating I throw-up the food.

(Central Ghana, pregnant woman 37 years old, three children)

In Malawi, HIV was also viewed as a provoking the illness that overlapped with biomedically defined malaria, "malungo".

I: Apart from mosquitoes and general body pains, are there other things that cause malungo?

R: What causes malungo is if you have HIV. You just see malungo frequently, so you wonder and you go to the hospital for a test to see how you are, whether you are ok. Then they find the virus, so you are aware that the malungo is a result of the HIV.

(Malawi. Group discussion with female community members)

Apart from in extreme circumstances, the local malarias and bouts of these illnesses during pregnancy were not associated with witchcraft at any of the sites. 


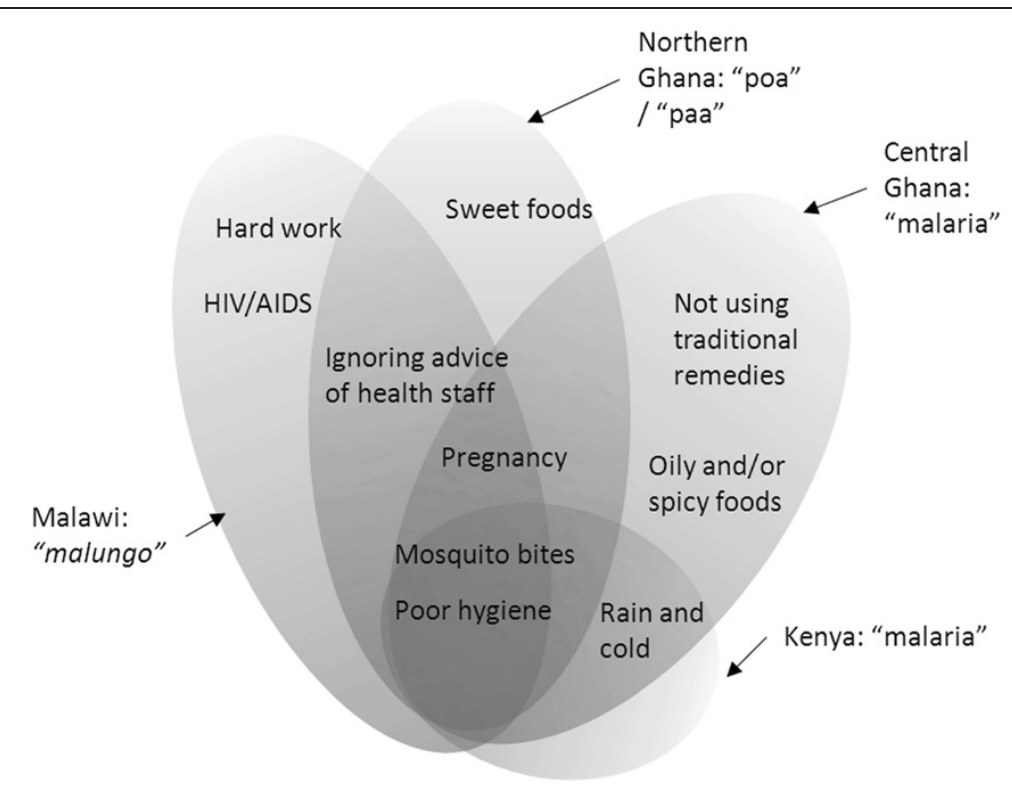

Figure 2 The overlapping causes of local malarias.

Vulnerability to malaria in the context of pregnancy risks At all the sites, pregnant women, along with children, were generally viewed as particularly vulnerable to malaria. Individually, however, women were more uncertain about their relative risk whether pregnant or not, and not all viewed themselves as more likely to suffer malaria when pregnant. Among the women with previous experience of malaria when pregnant or not, the bouts during pregnancy were generally considered to be more severe, but there were exceptions, for whom experiences of severe malaria outside of pregnancy had led them to consider it worse than their experiences when pregnant.

The results of the free listing and sorting exercises (Table 2) showed that the illnesses that overlapped with biomedically defined malaria were amongst the most cited problems: the most cited in Kenya, Malawi and northern Ghana, and third most cited in central Ghana. There was a clear consensus across the sites that malaria was the most dangerous problem for pregnant women. Malaria was also considered frequent during pregnancy at all the sites except central Ghana. The lesser relevance of the locally defined illnesses in the narratives of pregnant women in central Ghana was indeed later confirmed during in-depth interviews: an important group of women, especially the youngest pregnant women, knew nothing about the disease and the majority reported never having experienced, whether pregnant, or not.

Vomiting, weakness and headache were often cited as problems for pregnant women across the sites, but were also associated with the various local malarias. Indeed, there was a reported overlap between the symptoms of pregnancy and those of mild bouts of malaria: headache, weakness, paleness, vomits, lack of appetite, fever and even "malaria" were considered possible symptoms of pregnancy. To differentiate between the symptoms as signs of pregnancy or bouts of malaria, respondents referred to the severity of symptoms.

I: How would a woman know that she's pregnant?

R: Her skin changes; she gets malaria; she sleeps a lot; she becomes lazy.

(Central Ghana. In-depth interview with an opinion leader)

Table 2 Health problems that pregnant women suffer (free listing and sorting)

\begin{tabular}{llccc}
\hline & Central Ghana & Northern Ghana & Malawi & Kenya \\
\hline Most cited & Vomiting & "Poa" / "Paa" & "Malungo" & "Malaria" \\
\cline { 2 - 5 } & Weakness & Vomiting & Vomiting & Backache \\
\cline { 2 - 5 } & "Malaria" & Weakness & "Meadache \\
\hline Considered dangerous & "Malaria" & "Poa" / "Paa" & "Malaria" \\
\cline { 2 - 5 } & Bleeding & Abdominal pain & Anaemia & "Lack of blood" \\
\cline { 2 - 5 } & Weakness & "Lack of blood" & Vomiting & Abdominal pain \\
\hline
\end{tabular}


I: So how do you differentiate between the signs of malungo and those that are just the signs associated with being pregnant?

R: We look at the seriousness of the disease; so you can tell that this is malungo because it is serious, or they are just signs associated with pregnancy because they are not so serious.

(Malawi. Group discussion with community women).

Pregnant women's greater risk of suffering one of the malarias was mostly attributed to their general vulnerability to disease: pregnant women are "soft", "weak", "prone to a number of things", "two in one", "carry something inside that means they always have a high temperature", etc. Women's inability to meet their dietary needs and multiple poorly spaced pregnancies were considered to compound the weakness of pregnant bodies. Although pregnant adolescents were not seen as particularly vulnerable to malaria, their social vulnerability was stressed during the interviews: dropping out of school, terminating the pregnancy (often clandestinely), being rejected by their families, and lacking economic resources were some of the risks mentioned for unmarried pregnant adolescents. Adolescents also had least knowledge of these and other diseases during pregnancy and - as was reported and observed - depended most on others (husbands/"the man who impregnated them", mothers and other relatives, husbands' family) to take care of their pregnancies.

\section{Effects}

In general, pregnant women and community members acknowledged that the malarias had generally negative effects on women's health during pregnant and that of their unborn children. If left untreated, they considered that the symptoms would remain, and that they posed a risk to the life of the pregnant women or the unborn child. Indeed, community members saw the association with death as the main reason for considering malaria to be the most serious problem during pregnancy. Similarly, miscarriage, stillbirths and preterm delivery were the most commonly mentioned consequence of the various malarias in Ghana and Malawi. In Kenya, this direct link, although less common, was not unheard of.

I: So before when we were talking you said that you had suffered malaria during your pregnancies - can you tell us what the symptoms were?

R: Headache, low blood and then my body was turning yellowish. So, when I came back after one week, I miscarried

I: You think the malaria led to the miscarriage?

R: Yes, I think so.

(Kenya. In-depth interview with a pregnant woman, 24 years old, one child)

Further probing about the causes of miscarriage illustrated that the local malarias were among many other potential causes and was not necessarily the most relevant (Figure 3). At all sites, it was considered that malaria and frequent sickness in general played a role, but vaginal bleeding, problems in the uterus, worries, hard work and falls during pregnancy were also important causes of miscarriage. Using bitter traditional or biomedical medicines was acknowledged as the most relevant cause of miscarriage in Ghana and Malawi. Indeed, at these sites, the link between self-medication with either traditional or biomedical drugs and miscarriage was a key message during the health talks given in health facilities when women attended ANC. Moreover, in northern Ghana, there were several reports of pregnant women who had been administered anti-malarials to treat malaria but had miscarried. They had however blamed the drugs and not the illness for the miscarriage. In more general terms, it was also reported that poor ANC attendance or ignoring the advice of ANC staff

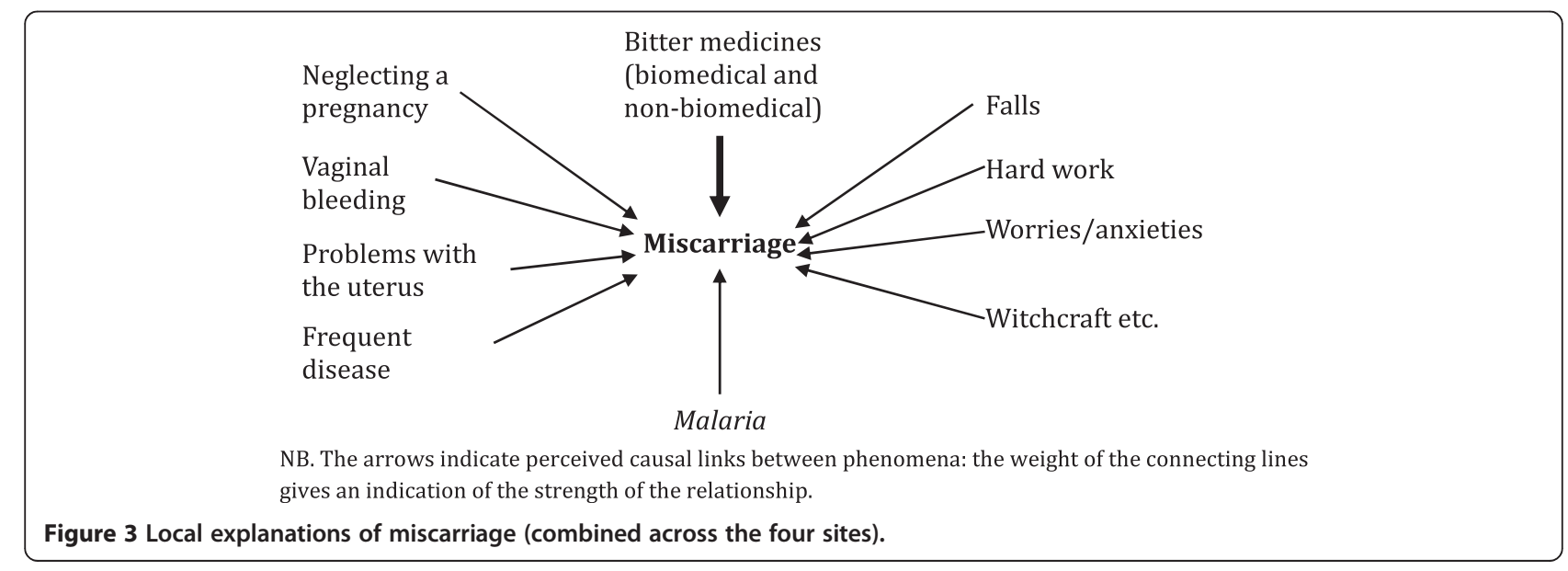


could lead to miscarriage. Witchcraft was often suspected to have caused a miscarriage, especially in the case of multiple miscarriages. In central Ghana, asram, a specific supernatural illness that attacked the babies though their mothers, was also cited as a cause of miscarriage.

Previous severe bouts of malaria suffered during pregnancy or by their infants prompted women to link them with anaemia/"lack of blood". Across all the sites, local terms were directly translated as "lack of blood", the condition which closely approximated the biomedically defined anaemia, in terms of symptoms, particularly with regard to paleness and lethargy. However, because there were some differences (as is detailed below), the italicized lack of blood, is used to differentiate local from biomedical understandings. For many informants, the link was so obvious that they would not mention it until prompted by the researchers, especially in central Ghana, where anaemia-associated paleness was considered one of the main symptoms of malaria amongst children and pregnant women. The causal link was however bidirectional: informants at all sites, particularly in Ghana and Malawi, regarded the local malaria as a cause of lack of blood largely because "fever dried blood" and to a lesser extent because "mosquitoes sucked" blood; others reported that bouts of malaria particularly affect women because they are weak as they have insufficient blood (though this was a vague link in Kenya).

The relationship between pregnancy and lack of blood was not restricted to the local malarias (Figure 4). Blood was rather considered to be a crucial element in pregnancy and foetal development: lack of blood was strongly related with the assertion that "pregnant women share the blood with the unborn child". It was also associated with hazards during delivery, because this is a moment when women "lose more blood". Women's diet also had implications for anaemia because food was seen as a key way of avoiding lack of blood. Women were said to need better nutrition during pregnancy, but respondents often described how poverty meant that they were often unable to meet this need. Furthermore, pregnancy-related symptoms, such as vomiting and lack of appetite which prevented women from eating, also contributed to this lack of blood. Moreover, during interactions with pregnant women as part of ANC, health workers emphasized the relevance of diet during pregnancy. In Malawi, the relationship between malaria, pregnancy and anaemia was further complicated by the connections that respondents made between lack of blood and HIV/AIDS.

I: What can account for a pregnant woman's lack of blood?

R: The child takes its blood from you so you need to eat a lot and healthily so that, when the child takes blood from you, you're able to stand the effect and not suffer lack of blood.

(Central Ghana. In-depth interview with a woman over 30 years old)

I: Is lack of blood common in pregnant women?

R: Pregnant women... it comes, and they have it. Even when the baby is sick, you hear that he has no blood. So where did the blood go? The problem is the mother. Her blood has a bad disease, which she has given the baby. She has bad blood.

I: Can you think about what causes lack of blood? R: HIV is destroying blood.

(Malawi. In-depth interview with a traditional birth attendant)

As previously mentioned, at all the sites, respondents reported that the unborn child was affected by malaria. However, only respondents in northern Ghana and in

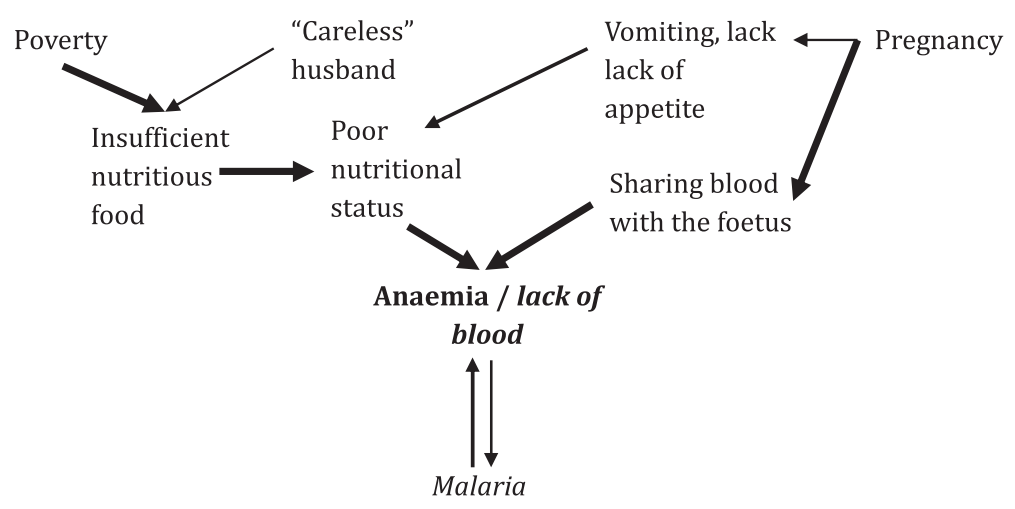

NB. The arrows indicate perceived causal links between phenomena: the weight of the connecting lines gives an indication of the strength of the relationship.

Figure 4 Local explanations of anaemia (combined across the four sites). 
Malawi frequently stated that the negative effects of local malarias on unborn children would later emerge as congenital malaria.

I: Let's suppose you have malaria and you did not take any medication, how could you be affected?

R: I might not get well.

I: What about the problems for the child you are expecting?

R: It could be born with malungo.

I: So, if born with malungo, what are the problems that the child might experience?

R: I might struggle to go to the hospital frequently with him or her.

(Malawi. In-depth interview with a pregnant woman,

17 years old, second pregnancy, childless)

Only two informants linked malaria and LBW: one midwife in northern Ghana, and one pregnant woman who was participating in a clinical trial. The weight of the baby was mainly related with nutrition during pregnancy, and thus lack of food, vomiting or lack of appetite and anaemia were considered relevant causal factors. Birth weight preferences were also discussed: in central Ghana and Malawi women preferred newborns to be big as this was considered a sign of health. Whereas, in northern Ghana, women tended to prefer smaller children, which would grow bigger once born, to have an easy delivery. In Kenya, although respondents recognized that babies with a LBW tended to suffer more problems, no particular size preference was stated.

\section{Risk during pregnancy}

The data illustrated multiple relationships between the local malarias, other health problems during pregnancy, such as anaemia and HIV/AIDS, and pregnancy itself. However, for the respondents, risk during pregnancy was not only restricted to bodily complaints: a range of social and financial problems emerged during the free listing and sorting exercises and interviews. Such problems cannot be isolated from biomedically (or locally) defined diseases because they have implications for the health of pregnant women. In the communities, poverty was considered to provoke lack of blood during pregnancy. As observed and reported by respondents, social relations with husbands and other family members were central to women's access to resources, and a source of stress, concern and worries that women viewed as possibly harming a pregnancy. Antagonistic relations with relatives or neighbours could also bring about abortions and problems during delivery through witchcraft.

The health implications of social problems were particularly pronounced amongst adolescents: for women of this age group, the consequences of social stigma linked to pregnancy were considered far more relevant than the risk of malaria. Furthermore, on some occasions, this stigma was associated with unsafe abortions and poor access to antenatal care.

I: Which types of problems do adolescents have when pregnant?

R: They will see some problems. For example, they cannot continue with school, they had sex with different people so they don't know the real father of the baby or they will see that their future has been messed up.

I: Which challenges do they face?

R: They can abort a pregnancy because they suffer a lot of stress $(. .$.

I: How do people in the village talk about pregnancy in adolescents?

R: People always disrespect them or they are less valued in the community.

(Kenya, pregnant woman case study, 24 years old, one child)

Health issues can also affect social relations. For a pregnant woman, an HIV diagnosis could have a critical impact on her marriage, as could recurrent pregnancy loss and infertility. The symptoms of pregnancy (malaria or lack of blood), such as weakness, (referred by many as "laziness") could make it difficult for women to work in the fields and this had both financial and relational consequences.

The people with whom you live might think that you deliberately don't want to work, but you know that it hurts. So he can say that he would not do what he usually does for you because you are being deliberately lazy. You know that the work is yours to do but you lie down and... you cannot do it.... They would say that you should get up and work so that your body is smart but you are lying down... You cannot get up. They say that you are doing it deliberately, that you have let the pregnancy overcome you.

(Northern Ghana. Pregnant woman, 27 years old, two children)

This problem appeared frequently during discussions, especially in northern Ghana, but the case studies showed that although women reduced their heavy household duties during pregnancy (such as collecting water and lifting other heavy weights), many continued with chores almost until delivery and then begun again weeks or days afterwards.

\section{Discussion}

Across the four sites, the various local malarias overlapped considerably with the biomedical concept of 
malaria. Nonetheless, with regard to symptoms, vulnerable groups, outcomes and aetiology, this overlap, in the context of pregnancy, was both site-specific and incomplete. This partial overlap meant that respondents occasionally identified an illness as malaria, but health professionals did not diagnose malaria. In other instances, health professionals might diagnose malaria, whereas the sufferer did not consider it to be malaria. The boundaries between local and biomedical concepts overlap and they are not clearly defined (this is represented in an abstract, visual manner in Figure 1), with symptoms varying between respondents and from one episode of illness to the next. In light of the uncertainty surrounding the symptoms of biomedically defined malaria during pregnancy [8,9], in Figure 1 the area that represents biomedically defined malaria is also poorly defined.

These findings resonate with previous qualitative research that has identified a variety of local malarias that overlap, often partially, with biomedically defined malaria and malaria during pregnancy [6,26,32-35]. In southern Malawi, previous studies employing qualitative methods at different research sites [35,36] have also described illness(es) that were referred to using the Chichewa term, "malungo". Moreover, these illnesses share some (but not all) characteristics with the "malungo" that respondents described during fieldwork in Malawi for this research: both were caused by hard work or mosquitoes and the severity varied, but, for example, in the previous studies, respondents viewed "malungo" during pregnancy as less dangerous. Within Malawi, the significance of the disease concept attached to this particular Chichewa term has therefore seemingly changed over time or it differs at these research settings (separated by hundreds of kilometres). This heterogeneity underlines how, when carrying out research (or implementing interventions) in the same or nearby sites over time or in other settings where the same terms are used, the meaning of such terms - in terms of the attached illness concepts - cannot be taken for granted; nor can such terms be unproblematically translated as malaria.

Respondents across all the sites considered the local illnesses that overlapped with biomedically defined malaria, to be the most dangerous disease during pregnancy and pregnant women were regarded as one of the most vulnerable groups. These findings coincide with previous studies in Zambia [37], Malawi [38], Tanzania [39], Ethiopia [40], Kenya [41], Uganda [34,35,42,43], Nigeria [44], Senegal [45] and the Gambia [46]. At all sites except central Ghana, across the different categories of respondent, the local malaria was viewed as common during pregnancy.

The relationship between local illness and biomedically defined malaria was however further complicated by women's experience of pregnancy. As other authors have highlighted $[26,32,33,35,42,45,46]$, many symptoms linked to malaria, including headache and fever, were considered to be normal symptoms or problems related to pregnancy. In some instances, pregnancy was also offered as an explanation for bouts of malaria. Furthermore, whether women labelled such episodes as about of Malaria depended upon the seriousness of symptoms (and their impact on their daily lives). Hence local malarias and pregnancy are interwoven domains of experience.

Local explanations of pregnancy loss, anaemia and LBW were complex and, in that sense, they resonate with the findings of biomedical research [14-20]. However, among their multiple causes, malarias were never considered the most important factor. Emphasis was also often placed on their social underpinnings, such as poverty, hard work during pregnancy, an unhelpful husband, or psychological strain and general ill health. Moreover, as previous anthropological research has emphasized $[47,48]$, the findings demonstrate the interrelation between social, economic and health problems during pregnancy.

\section{Framework for MiP interventions}

Given the complex relationships between experiences of pregnancy on the one hand and malaria/malaria on the other, ANC must address the web of pregnancy-related problems that women often perceive as interrelated. It is therefore essential that WHO-recommended procedures for ANC [49] promoting an integral care strategy are followed and that the fragmentation of ANC into in multiple isolated procedures (as is sometimes perceived in practice) is avoided.

Comprehensive ANC is essential to improve safe motherhood and ensure effective malaria prevention and control, but other social and economic strategies are also needed to fully address pregnant women's vulnerabilities. This is especially relevant in the case of adolescents, for whom the social risks of pregnancy eclipse their higher vulnerability to, and greater morbidity and mortality as a result of, MiP. The social consequences and the stigma of adolescent pregnancy must also be addressed to ensure ANC attendance and access to MiP interventions $[7,35,50]$.

\section{Health messages about malaria and MiP}

The results show that some messages about malaria and pregnancy are nowadays part of local ideas about health and disease: mosquitoes are recognized as the main cause of malaria at all the sites; lack of hygiene is considered an important cause of disease, even though it is sometimes viewed as a cause of malaria [23]; women are aware that they should consume nutritious food to prevent anaemia during pregnancy; and self-medication is often viewed as a 
risky practice that can provoke miscarriage. Nonetheless, the relevant health messages, and the form in which they are communicated, could be modified to promote greater awareness of MiP and its consequences. Two specific areas seem particularly relevant:

1. Awareness of MiP's deleterious effects was not universal: miscarriage and congenital malaria were the best known; anaemia was acknowledged by some; but LBW was not mentioned as a direct effect. Consequently, it is important to move from a generic model of the severity of MiP to more pragmatic and specific messages about malaria's serious implications during pregnancy, focusing on its role in compounding or provoking anaemia, LBW and pregnancy loss.

2. At all the sites, adolescents' greater risk of MiP was relatively unknown. A broader approach is however necessary to ensure the effective communication of messages about adolescent's vulnerability to MiP. To reach adolescent before they become pregnant requires incorporating such messages into health education at school. However, given their dependence on other people to access ANC, targeting health messages at adolescents' is not sufficient: entire communities must rather be aware of these messages and take responsibility for the success of health promotion.

\section{Site specificities and their implications}

Other interventions and health messages require tailoring to local settings. In central Ghana, it is important that women learn to identify MiP and raise their awareness that it is a common problem during pregnancy. On the other hand, the prominence of weakness and paleness as malaria symptoms reveals a close link between anaemia and malaria that can facilitate specific messages regarding the repercussions of $\mathrm{MiP}$ (as described in other studies conducted in a nearby area [49]).

In northern Ghana, health providers should be aware of the local links between malaria and sweet-tasting foods, and local preferences for delivering LBW babies in order to avoid misunderstandings and negative reactions to their advice. The association of malaria with consuming sweet-tasting foods and drinks during pregnancy is a source of misunderstanding. Some women distrust and therefore ignore nurses' advice about eating something sweet in order to avoid the side effects of malaria treatment.

In an earlier article on the possible contributions from the social sciences to research on malaria in pregnancy, the authors cite an example from Burkina Faso of women who prefer "the baby to grow after giving birth instead of before" and thus having bigger babies could be considered an undesired effect of malaria treatment [51]. During data collection, a similar preference for delivering small babies was only identified in the neighbouring northern Ghana. This preference needs to be addressed because it probably interferes with some of the advice regarding healthy nutrition. Nevertheless, given the lack of association between LBW and local malaria concepts, it does not seem to affect acceptance of MiP treatment or prevention.

In Malawi, there were associations between perceptions of HIV and malaria that need to be taken into account for health campaigns. HIV interacts with and complicates malaria's epidemiology and pathology $[1,52,53]$ as well as the social context and understanding of disease during pregnancy. The shared understanding of malaria and HIV could, in part, explain the common association between $\mathrm{MiP}$ and congenital malaria, which, given its rare symptomatic presentation, is notable [12]. It is related to local understanding of illness transmission during pregnancy, which, in Malawi, has probably been reinforced by the widespread health education on the prevention of motherto-child HIV transmission.

\section{Strengths and limitations}

The use of qualitative methods, in combination with longterm data collection, has enabled the analysis of understandings of MiP and their contextualization within a background of pregnancy-related problems. The comparative approach, using parallel techniques and topics of research, also drew attention to both the similarities and differences between the sites, which could have otherwise been taken for granted.

This article has presented no specific data on health professionals' views of MiP their perceptions of MiP require further analysis because, as they are instrumental to the supply of effective MiP interventions. Finally, the inclusion of an additional site with low seasonal malaria transmission would have enabled greater assessment of how the epidemiological context influences local perceptions of MiP.

\section{Conclusion}

At each study site, complex relationships between local concepts of malaria that overlapped with biomedically defined malaria and the symptoms of pregnancy were identified. Pregnancy was often considered to provoke malaria; malaria was also seen as a symptom of pregnancy; and many of the symptoms of malaria and pregnancy overlap. Treatment and prevention for MiP should therefore be delivered as part of a comprehensive ANC programme and fragmentation of ANC into separate disease-specific interventions avoided. However, it is important to avoid expanding MiP interventions to the 
detriment of other core health issues, such as anaemia and miscarriage, some of which can be linked to MiP but whose aetiologies are more complex. This study also underlines how when designing MiP prevention and control strategies, in addition to the changing contexts of malaria transmission and drug resistance, it is necessary to take into account the specific and fluid local relationships between malaria and illness during pregnancy. These relationships are especially relevant for the design of adequate health education that should form part of the promotion of any new health intervention.

\section{Competing interests}

The authors declare that they have no competing interests.

\section{Authors' contributions}

AM contributed to the overall study design; supervised and assisted with data collection in Ghana and Malawi; analysed the data from these sites; prepared the first draft of the manuscript and contributed to its revision based on comments from co-authors. CP contributed to the overall study design; supervised and assisted with data collection in Kenya; analysed the data from this site; provided comments on the first draft of the manuscript and contributed to its revision based on the comments from co-authors. LM collected data at the Malawi site; revised the manuscript and provided comments. SC collected and supervised data collection in northern Ghana; revised the manuscript and provided comments. NAA collected data in central Ghana; revised the manuscript and provided comments. FW collected data in Kenya; revised the manuscript and provided comments. AH supervised data collection in northern Ghana; revised the manuscript and provided comments. PO supervised data collection in Kenya; revised the manuscript and provided comments. LK supervised data collection in Malawi; revised the manuscript and provided comments. HT supervised data collection in central Ghana; revised the manuscript and provided comments. RP conceived and designed study; obtained project funding; provided comments and contributed to the revision of the manuscript based on comments from all co-authors. All authors read and approved the final version of the manuscript.

\section{Acknowledgements}

The authors would like to thank the respondents who participated in the programme of research at each site and took time to share their experiences and opinions with members of the research team. We would like to express our gratitude to Lianne Straus who was instrumental in the early phases of setting up the programme of research and to the large teams of fieldworkers who participated in data collection in Ghana and Malawi: Charity Siayire, Louis Alatinga, Dominic Anaseba, Gertrude Nyaaba and Gideon Lugunia in northern Ghana; Collins Zamawe, Chikondi Kwalimba, Alinafe Chibwana and Blessings N Kaunda in Malawi. Our thanks to Mary Hamel who made a key contribution to setting up the study in Kenya, and to Denise Roth Allen, and Pascal Magnussen for the comments that they provided on a previous version of the article.

The publication is supported and endorsed by the MiP Consortium, which is funded through a grant from the Bill and Melinda Gates Foundation to the Liverpool School of Tropical Medicine (www.gatesfoundation.org), Grant OPP46099. The funders had no role in study design, data collection and analysis, decision to publish, or preparation of the manuscript.

\section{Author details}

${ }^{1}$ Centre de Recerca en Salut Internacional de Barcelona (CRESIB), Hospital Clínic-Universitat de Barcelona, Barcelona, Spain. ${ }^{2}$ Departamento de Antropología Social, Universidad Complutense de Madrid, Madrid, Spain. ${ }^{3}$ Centre for Social Science and Global Health, University of Amsterdam, Amsterdam, The Netherlands. ${ }^{4}$ College of Medicine, University of Malawi, Blantyre, Malawi. ${ }^{5}$ Navrongo Health Research Centre, Navrongo, Ghana. ${ }^{6}$ Department of Community Health, School of Medical Sciences, Kwame Nkrumah University of Science and Technology, Kumasi, Ghana. ${ }^{7}$ The Kenya Medical Research Institute (KEMRI) and Centers for Disease Control and Prevention (CDC) Research and Public Health Collaboration, Kisumu, Kenya.
Received: 7 May 2013 Accepted: 17 July 2013

Published: 22 July 2013

\section{References}

1. Desai M, ter Kuile FO, Nosten F, McGready R, Asamoa K, Brabin B, Newman RD: Epidemiology and burden of malaria in pregnancy. Lancet Infect Dis 2007, 7:93-104.

2. World Health Organization: A strategic framework for malaria prevention and control during pregnancy in the African region, World Health Organization Regional Office for Africa. Brazzaville: Republic of the Congo; 2004.

3. Morris K: Filling the gaps to tackle malaria in pregnancy. Lancet Infect Dis 2011, 11:167-168.

4. van Eijk AM, Hill J, Alegana VA, Kirui V, Gething PW, ter Kuile FO, Snow RW Snow RW: Coverage of malaria protection in pregnant women in subSaharan Africa: a synthesis and analysis of national survey data. Lancet Infect Dis 2011, 11:190-207.

5. Malaria in Pregnancy Consortium. [www.mip-consortium.org/]

6. Pell C, Straus L, Andrew EWW, Meñaca A, Pool R: Social and cultural factors affecting uptake of interventions for malaria in pregnancy in Africa: a systematic review of the qualitative research. PLoS One 2011, 6:e22452

7. Pell C, Meñaca A, Were F, Afrah NA, Chatio S, Manda-Taylor L, Hamel MJ, Hodgson A, Tagbor $\mathrm{H}$, Kalilani L: Factors affecting antenatal care attendance: results from qualitative studies in Ghana. Kenya and Malawi. PLoS One 2013, 8:e53747.

8. Tagbor H, Bruce J, Browne E, Greenwood B, Chandramohan D: Malaria in pregnancy in an area of stable and intense transmission: is it asymptomatic? Trop Med Int Health 2008, 13:1016-1021.

9. Bardají A, Sigauque B, Bruni L, Romagosa C, Sanz S, Mabunda S, Mandomando I, Aponte J, Sevene E, Alonso PL: Clinical malaria in African pregnant women. Malar J 2008, 7:27.

10. Rogerson SJ, Hviid L, Duffy PE, Leke RFG, Taylor DW: Malaria in pregnancy: pathogenesis and immunity. Lancet Infect Dis 2007, 7:105-117.

11. Guyatt $\mathrm{HL}$, Snow RW: Impact of malaria during pregnancy on low birth weight in sub-Saharan Africa. Clin Microbiol Rev 2004, 17:760-769.

12. Hartman TK, Rogerson SJ, Fischer PR: The impact of maternal malaria on newborns. Ann Trop Paediatr Int Child Health 2010, 30:271-282.

13. Malhotra I, Mungai P, Muchiri E, Kwiek JJ, Meshnick SR, King CL: Umbilical cord-blood infections with Plasmodium falciparum malaria are acquired antenatally in Kenya. J Infect Dis 2006, 194:176-183.

14. Ouédraogo S, Koura GK, Accrombessi MMK, Bodeau-Livinec F, Massougbodji A, Cot M: Maternal anemia at first antenatal visit: prevalence and risk factors in a malaria-endemic area in Benin. Am J Trop Med Hyg 2012, 87:418-424.

15. Brabin BJ, Hakimi M, Pelletier D: An analysis of anemia and pregnancyrelated maternal mortality. J Nutr 2001, 131:604S-615S.

16. van Broek d, Nynke R, Letsky EA: Etiology of anemia in pregnancy in south Malawi. Am J Clin Nutr 2000, 72:247s-256s.

17. Buttha ZA, Yakoob MY, Lawn JE, Rizvi A, Friberg IK, Weissman E, Buchmann E, Goldenberg RL: Stillbirths: what difference can we make and at what cost? Lancet 2011, 377:1523-1538.

18. Buttha ZA, Ahmed T, Black RE, Cousens S, Dewey K, Giugliani E, Haider BA, Kirkwood B, Morris SS, Sachdev HPS, Shekar M: What works? Interventions for maternal and child undernutrition and survival. Lancet 2008, 371:417-440.

19. Adjuik M, Smith T, Clark S, Todd J, Garrib A, Kinfu Y, Kahn K, Mola M, Ashra A, Masanja H: Cause-specific mortality rates in sub-Saharan Africa and Bangladesh. Bull World Health Organ 2006, 84:181-188.

20. Lawn JE, Cousens S, Zupan J: Lancet Neonatal Survival Steering Team: 4 million neonatal deaths: When? Where? Why?. Lancet 2005, 365:891-900

21. Heggenhougen HK, Hackethal V, Vivek P: The behavioural and social aspects of malaria and its control: an introduction and annotated bibliography. Geneva, Switzerland: World Health Organization; 2003.

22. Nichter M: Global health: Why cultural perceptions, social representations, and biopolitics matter: Arizona. USA: University of Arizona Press; 2008.

23. Hausmann Muela S, Muela Ribera J, Mushi AK, Tanner M: Medical syncretism with reference to malaria in a Tanzanian community. Soc Sci Med 2002, 55:403-413.

24. Pool R: Dialogue and interpretation of illness. Conversations in a Cameroon village. Providence: Berg Publishers; 1993.

25. Littlewood R(Ed.): On knowing and not knowing in the anthropology of medicine. California: Left Coast Press: Walnut Creek; 2007. 
26. Good BJ: The heart of what's the matter. The semantics of illness in Iran. Cult Med Psychiatry 1977, 1:25-58.

27. Ghana Statistical Service - Office of the President: Ghana - Population and Housing Census 2000. Accra, Ghana: Ghana Statistical Service - National Data Archive; 2000

28. Smith LA, Jones C, Adjei RO, Antwi GD, Afrah NA, Greenwood B, Chandramohan D, Tagbor $\mathrm{H}$, Webster J: Intermittent screening and treatment versus intermittent preventive treatment of malaria in pregnancy: user acceptability. Malar J 2010, 9:18.

29. Oduro AR, Wak G, Azongo D, Debpuur C, Wontuo P, Kondayire F, Welaga P, Bawah A, Nazzar A, Williams J: Profile of the Navrongo Health and Demographic Surveillance System. Int J Epidemiol 2012, 41:968-976.

30. Msyamboza K, Savage E, Kalanda G, Kazembe P, Gies S, D'alessandro U, Brabin BJ: Trends in pregnancy outcomes in Malawian adolescents receiving antimalarial and hematinic supplements. Acta Obstet Gynecol Scand 2010, 89:1011-1016.

31. Adazu K, Lindblade KA, Rosen DH, Odhiambo F, Ofware P, Kwach J, Van Eijk AM, Decock KM, Amornkul P, Karanja D: Health and demographic surveillance in rural western Kenya: a platform for evaluating interventions to reduce morbidity and mortality from infectious diseases. AmJTrop Med Hyg 2005, 73:1151-1158.

32. Winch PJ, Makemba AM, Kamazima SR, Lurie M, Lwihula GK, Premji Z, Minjas JN, Shiff CJ: Local terminology for febrile illnesses in Bagamoyo District, Tanzania and its impact on the design of a community-based malaria control programme. Soc Sci Med 1996, 42:1057-1067.

33. Launiala A, Kulmala T: The importance of understanding the local context: women's perceptions and knowledge concerning malaria in pregnancy in rural Malawi. Acta Trop 2006, 98:111-117.

34. Kengeya-Kayondo JF, Seeley JA, Kajura-Bajenja E, Kabunga E, Mubiru E, Sembajja F, Mulder DW: Recognition, treatment seeking behaviour and perception of cause of malaria among rural women in Uganda. Acta Trop 1994, 58:267-273.

35. Mbonye AK, Neema S, Magnussen P: Preventing malaria in pregnancy: a study of perceptions and policy implications in Mukono district, Uganda. Health Policy Plan 2006, 21:17-26.

36. Helitzer-Allen DL, Kendall C: Explaining differences between qualitative and quantitative data: a study of chemoprophylaxis during pregnancy. Health Educ Behav 1992, 19:41-54.

37. NetMark: NetMark formative qualitative research on insecticide treated materials (ITMs) in Zambia. Washington DC, USA: Academy for Educational Development; 2001

38. Tolhurst R, Theobald S, Kayira E, Ntonya C, Kafulafula G, Nielson J, Van Den Broek N: 'I don't want all my babies to go to the grave': perceptions of preterm birth in Southern Malawi. Midwifery 2008, 24:83-98.

39. Mubyazi G, Bloch P, Kamugisha M, Kitua A, ljumba J: Intermittent preventive treatment of malaria during pregnancy: a qualitative study of knowledge, attitudes and practices of district health managers, antenatal care staff and pregnant women in Korogwe District. North-Eastern Tanzania. Malar J 2005, 4:31.

40. Deressa W, Ali A: Malaria-related perceptions and practices of women with children under the age of five years in rural Ethiopia. BMC Publ Health 2009, 9:259.

41. Chuma J, Okungu V, Ntwiga J, Molyneux C: Towards achieving Abuja targets: identifying and addressing barriers to access and use of insecticides treated nets among the poorest populations in Kenya. BMC Publ Health 2010, 10:137.

42. Mbonye AK, Neema S, Magnussen P: Treatment-seeking practices for malaria in pregnancy among rural women in Mukono District, Uganda. J Biosoc Sci 2006, 38:221-237.

43. NetMark: NetMark formative qualitative research on insecticide treated materials (ITMS) in Uganda. Washington DC, USA: Academy for Educational Development; 2001.

44. NetMark: NetMark formative qualitative research on insecticide treated materials (ITMs) in Nigeria. Washington DC, USA: Academy for Educational Development; 2001.

45. NetMark: NetMark formative qualitative research on insecticide treated materials (ITMS) in Senegal. Washington DC, USA: Academy for Educational Development; 2001.

46. Brabin L, Stokes E, Dumbaya I, Owens S: Rural Gambian women's reliance on health workers to deliver sulphadoxine-pyrimethamine as recommended intermittent preventive treatment for malaria in pregnancy. Malar J 2009, 8:25.

47. Chapman RR: Endangering safe motherhood in Mozambique: prenatal care as pregnancy risk. Soc Sci Med 2003, 57:355-374.

48. Launiala A, Honkasalo M: Malaria, danger, and risk perceptions among the Yao in rural Malawi. Med Anthropol Q 2010, 24:399-420.

49. World Health Organization: Recommended interventions for improving maternal and newborn health. Geneva, Switzerland: World Health Organization; 2010

50. Peeters Grietens K, Gies S, Coulibaly SO, Ky C, Somda J, Toomer E, Muela Ribera J, D'Alessandro U: Bottlenecks for high coverage of intermittent preventive treatment in pregnancy: the case of adolescent pregnancies in rural Burkina Faso. PLoS One 2010, 5:e12013.

51. Muela Ribera J, Hausmann-Muela S, D'Alessandro U, Peeters Grietens K: Malaria in pregnancy: What can the social sciences contribute? PLoS Med 2007, 4:e92.

52. Flateau C, LeLoup G, Pialoux G: Consequences of HIV infection on malaria and therapeutic implications: a systematic review. Lancet Infect Dis 2011, 11:541-556

53. Brentlinger PE, Behrens $C B$, Micek MA: Challenges in the concurrent management of malaria and HIV in pregnancy in sub-Saharan Africa. Lancet Infect Dis 2006, 6:100-111.

doi:10.1186/1475-2875-12-257

Cite this article as: Menaca et al:: Local illness concepts and their relevance for the prevention and control of malaria during pregnancy in Ghana, Kenya and Malawi: findings from a comparative qualitative study. Malaria Journal 2013 12:257.

\section{Submit your next manuscript to BioMed Central and take full advantage of:}

- Convenient online submission

- Thorough peer review

- No space constraints or color figure charges

- Immediate publication on acceptance

- Inclusion in PubMed, CAS, Scopus and Google Scholar

- Research which is freely available for redistribution 